C-reactive protein (CRP) and muscle-associated enzymes creatine phosphokinase (CK) and lactate dehydrogenase (LD) were measured by routine laboratory methods.

Results In patients with PM, serum levels of S100A4 protein were significantly higher than those observed in healthy controls or DM patients $(148.6 \pm 351.5$ versus $80.75 \pm 285.1, p<0.01$ and $43.55 \pm$ $53.03, \mathrm{p}<0.05$, respectively). No significant differences in S100A4 levels were found between CAM patients $(119.9 \pm 414.0)$ and healthy controls or other myositis patients. In the whole group of IIM patients, serum S100A4 levels correlated with MYOACT score $(\mathrm{r}=0.39 ; \mathrm{p}<0.001)$ and its components Constitutional Disease Activity (DA) $(r=0.34 ; \mathrm{p}<0.001)$ and Pulmonary DA $(r=0.44$; $p<0.001)$. Serum S100A4 correlated also with Muscular DA $(r=0.25 ; p<0.05)$, CK $(r=0.33 ; p<0.01)$ and LD $(r=0.40$; $p<0.001)$. S100A4 levels correlated with Cutaneous DA ( $r=0.46$; $\mathrm{p}<0.01$ ) in DM patients and with Extramuscular Global Assessment only in PM patients $(r=0.55 ; \mathrm{p}<0.001)$. No significant correlations of S100A4 serum levels in patients with CAM were found. In the 11 longitudinal IIM samples there was no significant decrease of S100A4 serum levels observed. Multiple regression of the whole IIM patients group showed significant association of S100A4 serum levels with Pulmonary DA $(\beta=0.369 ; p<0.01)$, LD $(\beta=0.345$; $p<0.01)$ and severity of dysphagia $(\beta=-0.250 ; p<0.05)$. In $P M$ patients, S100A4 levels were associated with Extramuscular Global Assessment $(\beta=0.552 ; \mathrm{p}<0.01)$ and in DM patients with MYOACT $(\beta=0.557 ; p<0.01)$ and $\operatorname{CRP}(\beta=0.391 ; p<0.05)$.

Conclusions This is the first study showing that circulating levels of S100A4 are associated with several features of IIM disease activity, particularly with extramuscular components. We did not find any association of S100A4 levels and cancer associated myositis. Further studies analysing bioactive form of S100A4 and the role of S100A4 in cancer associated myositis are needed.

\section{A4.5 DO HIGH MOLECULAR WEIGHT ADIPONECTIN LEVELS ASSOCIATE WITH RADIOGRAPHIC PROGRESSION IN EARLY RHEUMATOID ARTHRITIS AND HAND OSTEOARTHRITIS?}

doi:10.1136/annrheumdis-2013-203217.5

IIR Klein-Wieringa, 'SN Andersen, 'JC Kwekkeboom, 'L Herb-v.Toorn, 'AHM van der Helm-van Mil, 2 I Meulenbelt, 'TWJ Huizinga, 'M Kloppenburg, 'REM Toes, ${ }^{1}$ A loan-Facsinay. 'Dept. of Rheumatology, Leiden University Medical Center, Leiden, The Netherlands; ${ }^{2}$ Dept. of Molecular Epidemiology, Leiden, The Netherlands

Background Adipose tissue can secrete many different soluble factors (adipokines) influencing whole body metabolism. Some of these adipokines, such as adiponectin, have been shown to influence radiographic progression in osteoarthritis $(O A)$ and rheumatoid arthritis (RA). In RA patients, total adiponectin (totAPN) levels in serum associate positively with radiographic progression, which suggestes an adverse effect on disease. Intruiguingly, in patients with hand OA, high totAPN levels in serum associated with a decreased relative risk for radiographic progression, suggesting a benificial effect on disease.

Adiponectin is a pleiotropic adipokine, which consists of several isoforms. Of these isoforms, high molecular weight adiponectin (hmwAPN) has been described as one of the most biologically active and its effect on radiographic progression in $\mathrm{RA}$ and hand $\mathrm{OA}$ is unknown.

Objective Therefore, we explored the possibility that the association between totAPN and disease progression is primarily mediated by the hmwAPN isoform.

Methods Concentrations of hmwAPN and totAPN were determined in baseline plasma of 324 RA patients from the Early Arthritis Cohort (EAC) and in baseline sera of 164 hand OA patients from the Genetics Arthrosis and Progression (GARP) study. The association between levels of hmwAPN and totAPN with radiographic progression were determined using a multivariate normal regression model (EAC cohort) or by generalised estimated equations (GARP cohort). Adjustments were made for age, gender, treatment strategy and Body Mass Index (BMI).

Results In RA patients totAPN associated positively with radiographic progression (Sharp van der Heijde scores) (association estimate $3.65, p=0.002$ ), whereas in patients with hand OA, totAPN associated negatively with radiographic progression (joint space narrowing (JSN)) (Odds 0.24/Odds 0.21, $p=0.002 / p=0.002$ two highest tertiles compared to the lowest tertile). HmwAPN on the other hand, did not associate significantly with radiographic progression in patients with hand OA or RA, although in patients with RA we did observe a trend towards a positive association (association estimate $1.53 p=0.07$ ) upon correcting for age, gender and treatment strategy. This trend was lost after further adjustment for BMI. Similar results were obtained when joint space narrowing (JSN) was used as outcome measurement.

Conclusions Our data further substantiate the connexion between APN-levels and radiographic progression in rheumatic disease and indicate that the differential effects associated between totAPN and radiographic progression in either in $\mathrm{RA}$ and hand $\mathrm{OA}$ is not mediated by (a selective effect of) hmwAPN.

\section{A4.6 EFFECTS OF CHOLIC ACID AND ITS DERIVATIVES IN EXPERIMENTAL ARTHRITIS}

doi:10.1136/annrheumdis-2013-203217.6

'Silvia Hayer, ${ }^{2}$ Emina Halilbasic, 'Birgit Niederreiter, 'Martin Willburger, 'Peter Mandl, 'Victoria Saferding, 'Stephan Blüml, 'Josef Smolen, ${ }^{2}$ Michael Trauner, 'Kurt Redlich. 'Medical University of Vienna, Department of Internal Medicine III, Division of Rheumatology; ${ }^{2}$ Medical University of Vienna, Department of Internal Medicine III, Division of Gasteroenterology

Introduction Bile acids play an important role in cholesterol metabolism and act as intestinal detergents for digestion and absorption of fats and fat-soluble vitamins. Disruption of bile flow causes cholestatic liver diseases. Derivatives of cholic acid (CA) such as nor-ursodeoxycholic acid (norUDCA) are promising therapeutic agents in the treatment of cholangiopathies. Previous studies also demonstrated anti-inflammatory and anti-fibrotic properties of norUDCA in experimental sclerosing cholestasis.

Objective To investigate the anti-inflammatory potential of CA and its derivatives ursodeoxycholic acid (UDCA) and nor-UDCA in in Collagen-induced arthritis (CIA), an animal model for inflammatory, erosive arthritis.

Methods Mice were prophylactically treated with CA, UDCA or nor-UDCA enriched diet pellets $(5 \mathrm{mg} / \mathrm{kg}$ diet $)$ or standard diet pellets (Placebo) ad libitum starting 1 week before the first immunisation with collagen. Animals were weekly assessed for clinical signs of arthritis, body weight and food consumption during the experimental period. After 10 weeks of treatment hind paws, liver, sera and lymph nodes were isolated for further analysis. Sera were investigated for anti-collagen antibodies, cytokine responses and liver parameters such as alkaline phosphatase (AP) and alanine transaminase (ALT). Paraffin-sections of hind paws were examined for histoptahological changes in synovial inflammation, subchondral bone erosion, cartilage damage and osteophyte formation. Cell populations within synovial pannus were identified by immunhistochemical stainings and were determined using HistoQuest software (from TissueGnostics)

Results Uptake of CA, UDCA and norUDCA was confirmed by serum analysis. Prophylactic treatment of CIA mice with UDCA and norUDCA could not significantly prevent disease incidence. In contrast, treatment with CA led to a marked increase in disease incidence and severity compared to Placebo treated animals. Whereas UDCA and norUDCA showed a similar course of clinical signs of 